\title{
Technè
}

La science au service de l'histoire de l'art et de la préservation des biens culturels

46 | 2018

Science et conservation

\section{La restauration du Christ en croix de la cathédrale Saint-Pierre de Nantes : redécouverte d'une technique de sculpture inédite grâce à la tomodensitométrie et aux micro-analyses}

The restoration of a Christ on the Cross in Nantes Cathedral: a little-known sculpture technique rediscovered thanks to CT scanning and microanalysis

Hélène Gruau, Marie-Pierre Etcheverry, Julie Guttierez et Yves Tillet

\section{OpenEdition \\ Journals}

Édition électronique

URL : http://journals.openedition.org/techne/370

DOI : $10.4000 /$ techne.370

ISSN : 2534-5168

Éditeur

C2RMF

Édition imprimée

Date de publication : 1 décembre 2018

Pagination : 44-56

ISBN : 978-2-11-152829-1

ISSN : 1254-7867

\section{Référence électronique}

Hélène Gruau, Marie-Pierre Etcheverry, Julie Guttierez et Yves Tillet, « La restauration du Christ en croix de la cathédrale Saint-Pierre de Nantes : redécouverte d'une technique de sculpture inédite grâce à la tomodensitométrie et aux micro-analyses », Technè [En ligne], 46 | 2018, mis en ligne le 19 décembre 2019, consulté le 23 juillet 2020. URL : http://journals.openedition.org/techne/370 ; DOI : https://doi.org/10.4000/techne.370

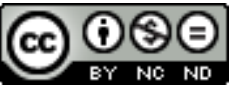

La revue Technè. La science au service de l'histoire de l'art et de la préservation des biens culturels est mise à disposition selon les termes de la Licence Creative Commons Attribution - Pas d'Utilisation Commerciale - Pas de Modification 4.0 International. 


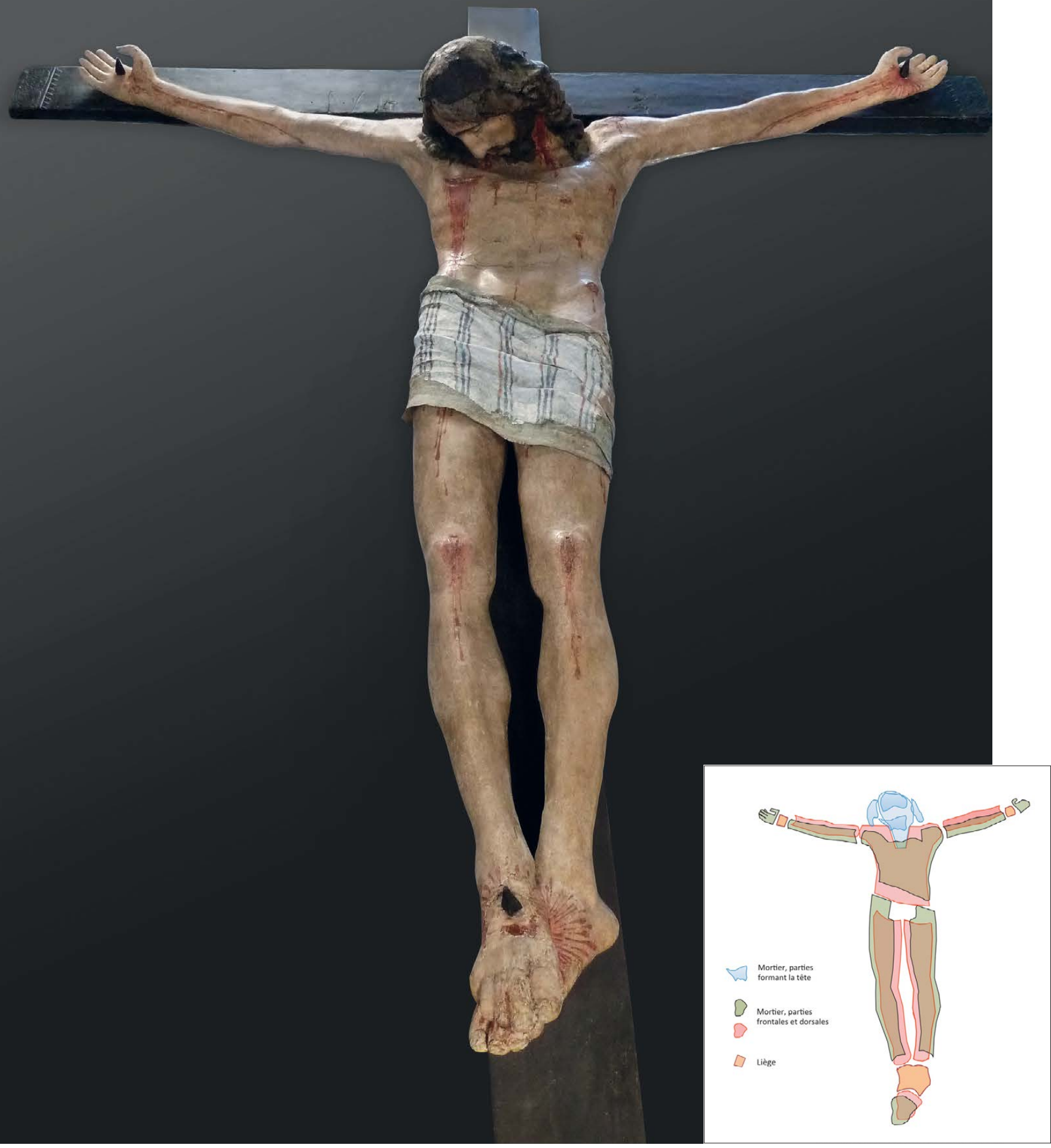

Fig. 1. Christ en croix, cathédrale Saint-Pierre de Nantes, fin XVI ${ }^{\mathrm{e}}$-début XVII ${ }^{\mathrm{e}}$ (Christ : $225 \times 202 \times 37 \mathrm{~cm}$ ).

Vue après restauration. (c) H. Gruau.
Fig. 2. Compréhension de la conception de l'œuvre à partir des investigations tomodensitométriques. @ H. Gruau. 
Hélène Gruau

Marie-Pierre Etcheverry

Julie Guttierez

Yves Tillet

\section{La restauration du Christ en croix de la cathédrale Saint-Pierre de Nantes : redécouverte d'une technique de sculpture inédite grâce à la tomodensitométrie et aux micro-analyses}

The restoration of a Christ on the Cross in Nantes Cathedral: a little-known sculpture technique rediscovered thanks to CT scanning and microanalysis.

Résumé. La cathédrale Saint-Pierre de Nantes conserve un Christ en croix de facture espagnole, daté de la fin du XVI ou du début du XVII siècle. C'est à l'occasion de sa restauration que cette ceuvre atypique appartenant au corpus des "sculptures légères " et aux cuvres de procession, proche des techniques de fabrication des Christ de maïs mexicains, a été redécouverte. Afin de recueillir des informations sur la technologie et les matériaux employés, plusieurs méthodes d'investigation assorties de micro-analyses approfondies ont été nécessaires. La réalisation d'une couverture tomodensitométrique détaillée a notamment été un élément décisif dans la compréhension des modes de construction originels et de l'état d'altération interne, et a démontré l'intérêt de cette technique d'imagerie performante pour les pratiques de restauration.

Mots-clés. Christ de procession, Christ de maïs mexicains, sculptures légères, art espagnol, polychromie, restauration, tomodensitométrie, micro-analyses $M E B$, mortier, textiles.
Abstract. Nantes Cathedral houses a Spanish Crucifixion dating from the late 16th or early 17th century. This atypical work, belonging to the group of "lightweight sculptures" and processional artefacts, closely related to the Mexican art of making sculptures of Christ from maize-stalk paste, was rediscovered during its restoration. In order to garner information about the technique and materials used, several methods of investigation and in-depth microanalyses were necessary. Producing a detailed CT scan image played a decisive role in understanding the original construction methods and internal alterations, and demonstrated the advantage of using this efficient imaging procedure in restoration practices.

Keywords. Processional Crucifix, Mexican maize-stalk sculptures of Christ, lightweight sculptures, Spanish art, polychromy, restoration, CT scanning, SEM analysis, binder, textiles.

\section{Un Christ espagnol à la cathédrale Saint-Pierre de Nantes}

Le Christ en croix de la cathédrale Saint-Pierre de Nantes (fig. 1), daté de la fin du XVI ${ }^{\mathrm{e}}$ ou du début du XviI ${ }^{\mathrm{e}}$ siècle et placé sur le pilier sud à l'entrée du chœur, était depuis plusieurs décennies nimbé de mystère : s'agissait-il d'une œuvre d'origine catalane comme le mentionnait un document d'archive appartenant à l'Association des Amis de la cathédrale $^{1}$ ? Était-ce une sculpture en papier mâché comme les conservateurs successifs se l'étaient imaginé ? Le ministère de la Culture - Direction régionale des affaires culturelles des Pays de la Loire procéda en 2009 à la dépose de l'œuvre dans le but de répondre à ces différentes questions, mais surtout afin d'établir un état sanitaire complet qui servirait ensuite de base à la définition d'un projet de restauration adapté à ce Christ très encrassé et fragilisé sur le plan structurel.
Un premier diagnostic fut donc confié à une restauratrice de sculptures ${ }^{2}$ qui découvrit le caractère composite et complexe de cette ouvre atypique. Un premier bilan radiographique a permis d'apprendre que celle-ci ne comportait pas de structure interne, mais les interrogations demeuraient entières quant à sa conception et la connaissance des matériaux constitutifs. Si nous ignorons encore tout des circonstances de l'arrivée de cette sculpture en France, cette première étude a permis en revanche de préciser son parcours jusqu'à l'édifice nantais : acheté au cours des années 1960 par le monastère Notre-Dame de Charité de Versailles, le Christ a ensuite été offert à l'Association des Amis de la cathédrale qui en fit don à l'État en 1977.

Hélène Gruau, restauratrice du patrimoine (hgruau@free.fr). Marie-Pierre Etcheverry, Dr-ingénieur archéomètre spécialisée en micro-analyse des matériaux du patrimoine, gérante de LAMOA Expertise à Bordeaux (contact.lamoa.exp@gmail.com). Julie Guttierez, conservatrice des monuments historiques, DRAC Pays de la Loire (julie.guttierez@culture.gouv.fr). Yves Tillet, Dr-HDR, directeur de recherches à l'INRA, directeur du service d'imagerie de la plateforme CIRE-UMR-PRC, Centre INRA Val-de-Loire (yves.tillet@inra.fr). 


\section{Choix des méthodes d'investigation pour la compréhension de la conception et de l'origine de ce Christ}

Le recours à plusieurs méthodes d'investigation impliquant l'imagerie tomographique puis la micro-analyse MEB (Microscopie électronique à balayage) s'est avéré indispensable suite à la première phase d'étude. La réalisation d'une couverture tomodensitométrique détaillée (effectuée par la plateforme CIRE du Centre INRA Val-de-Loire à Nouzilly, Indre-et-Loire) a été une étape décisive dans la compréhension des modes de construction originels, de l'état d'altération interne, et montre tout l'intérêt de cette technique d'imagerie performante pour les pratiques de restauration. Développée en clinique, elle s'appuie sur la mesure de l'atténuation d'un faisceau de rayons X après leur passage à travers la matière. L'objet à analyser est placé au centre d'une couronne contenant la source émettrice de rayons X et, diamétralement à l'opposé, se trouvent les détecteurs des rayons X qui vont mesurer le rayonnement résiduel. L'ensemble émetteur et détecteurs tourne autour du sujet afin d'enregistrer un très grand nombre d'images sous différentes incidences. Ces images sont ensuite analysées et utilisées pour reconstruire le volume général. En outre, avec les outils informatiques adaptés, il est possible de réaliser des coupes virtuelles et d'observer l'objet sous différents angles y compris de l'intérieur, un point de vue particulièrement pertinent dans le cas présent. Pour cette étude ${ }^{3}$, nous avons utilisé un tomodensitomètre Somatom ${ }^{\circledR}$ Siemens de 128 coupes permettant une résolution spatiale de $300 \mu \mathrm{m}$, et dont le tunnel de $78 \mathrm{~cm}$ de diamètre a permis d'explorer les différents éléments de la sculpture en un minimum d'acquisitions. Avec les variations de densités, des détails extrêmement précis ont pu être identifiés, tels que la structure des divers matériaux, les couches picturales au plomb, les cavités et les microfissures au sein des matériaux. Cette méthode a donné des informations capitales pour comprendre la conception et l'organisation de l'œuvre (fig. 2).

\section{La construction de la sculpture (planche 1)}

La conception du Christ de Nantes résulte en premier lieu de l'estampage d'un mortier dans une matrice. Les éléments constitutifs sont conçus séparément puis assemblés avant de recevoir la polychromie. L'œuvre est entièrement creuse (fig. 3) ; le tronc, les bras et les jambes sont formés de deux parties superposées, provenant de moules sans contredépouille et donc a priori réutilisables, les coutures ou zones de jonction entre les volumes obtenus se situant sur les côtés. La tête résulte de la combinaison de cinq parties au minimum ${ }^{4}$, probablement découpées dans le mortier frais, puis assemblées à plat joint et réunies au moyen de cordelettes passées dans des percements et bloquées par des microchevilles en bois.

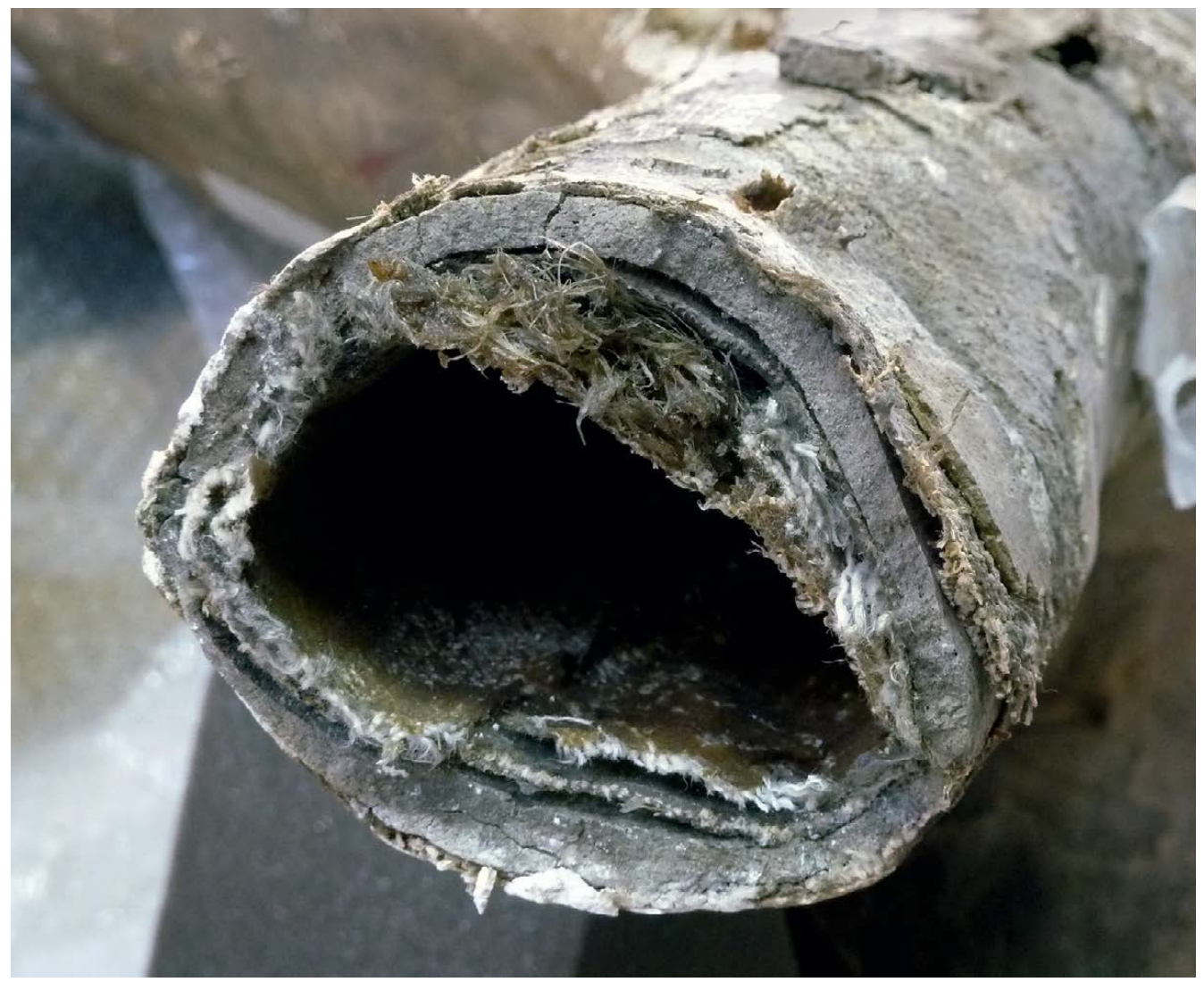

Fig. 3. Vue de l'organisation des matériaux au niveau de la cassure du bras droit. (c) H. Gruau. 

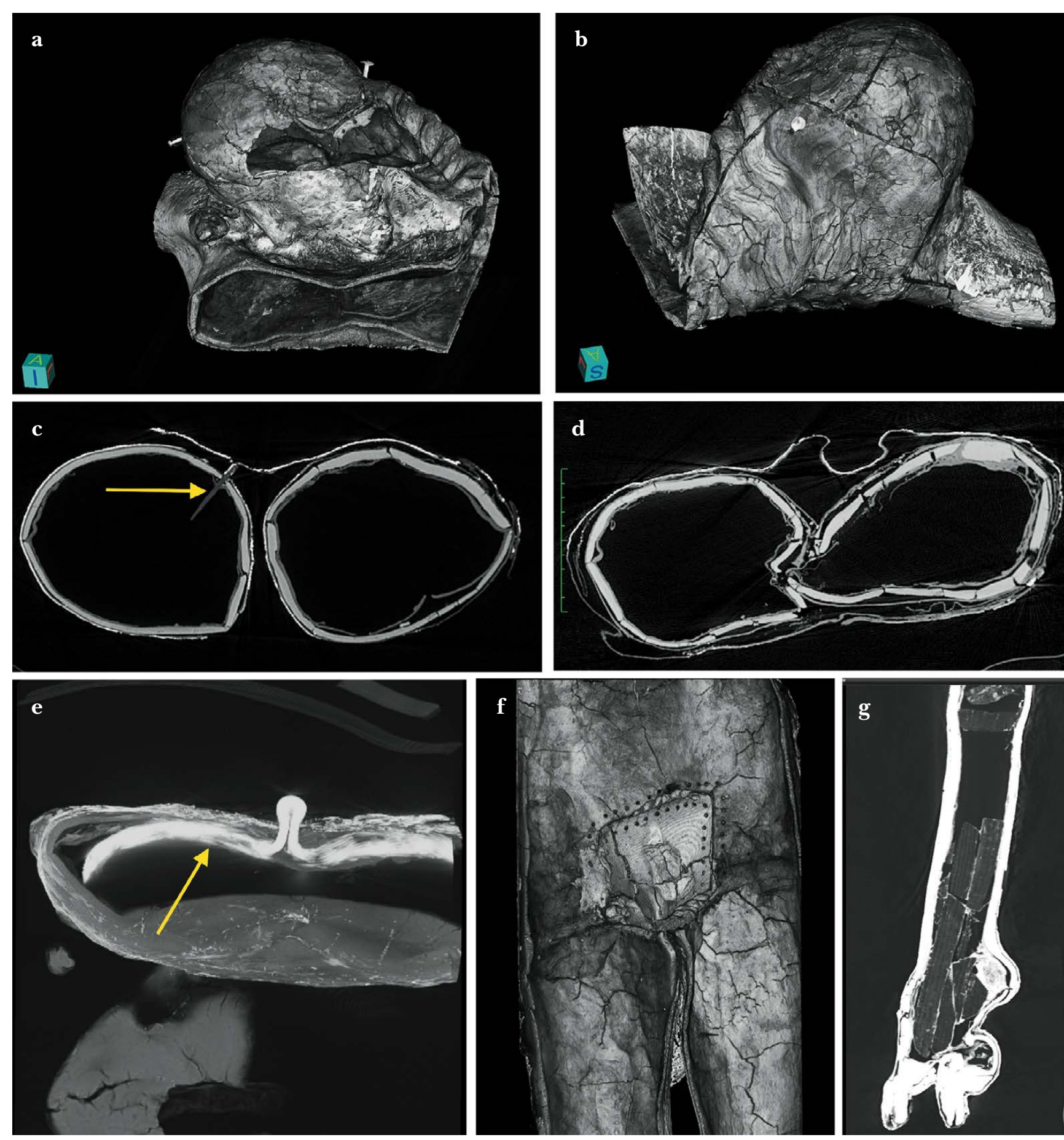

Planche 1. a et b. Vues 3D de la tête et de l'assemblage des parties constitutives en mortier ; c. Coupe au niveau des jambes et du perizonium, détail de l'aiguille en bois retenant le textile contre la jambe ; d. Coupe au niveau des jambes montrant l'état de fragmentation du mortier ; e. Coupe au niveau du mode de fixation original sur la croix (barre de cuivre insérée dans le mortier) ; f. Vue 3D de la trappe d'accès au niveau du bassin ; g. Coupe au niveau des pieds : fragments de liège internes. @ ILIAD3 Univ. Tours/B. Serres. 
L'assemblage des parties moulées se fait par application de fragments de textiles encollés (colle animale) sur la face interne, de qualité et taille variables, et mis en place de façon désordonnée, en fonction des besoins. L'encollage est très irrégulier, très fin ou épais et formant une couche brune, rigide et cassante. Les éléments ainsi obtenus sont ensuite associés par le même procédé de textiles encollés. Deux trappes sont aménagées afin de finaliser la construction : l'une à la jonction jambe tronc sous le perizonium, l'autre au dos de la chevelure, permettant de réunir bras, tête et torse. Ces trappes sont ensuite fermées par un tissu recouvert d'une couche de mortier, cousu dans le cas de la trappe sous le perizonium.

Toutes les observations du mortier nous montrent un matériau très marqué par les réseaux de fentes, le feuilletage et parfois la déformation des volumes initiaux. Il est important de constater que ces particularités sont inhérentes à la mise en œuvre ; la cohésion actuelle de la sculpture est obtenue dès l'origine grâce à la présence des textiles encollés, qui sont la véritable armature de l'ensemble.

Le volume obtenu est retravaillé : ajout de mortier sur la chevelure et certaines zones de jonction des parties constitutives, retrait de matière sur la grande plaie du flanc gauche, et peut-être sur certaines parties du visage afin de redonner un aspect plus vif aux détails. Mains et pieds sont mis en place : constitués d'une âme en liège permettant le passage des clous de la crucifixion, ils sont ensuite recouverts de mortier puis de fragments de textiles.
Le corps ainsi constitué est revêtu d'une nouvelle couche de tissus qui complète la structure de l'œuvre et permet l'application de la polychromie. Le perizonium est alors inséré : élément textile conçu séparément, il est appliqué humide et imprégné de colle autour de la taille du Christ de manière à réaliser un drapé naturel ${ }^{5}$. Il devait être noué sur la hanche gauche, partie aujourd'hui manquante. Quelques éléments supplémentaires viennent finaliser la conception : des clous en métal forgé sont placés dans la tête afin de recevoir la couronne d'épines ${ }^{6}$, et une aiguille en bois traversant le perizonium et le mortier de la jambe gauche permet le maintien du vêtement contre le corps. La sculpture est alors prête à recevoir la polychromie.

\section{Étude approfondie du mortier}

Le mortier grisâtre formant l'enveloppe corporelle s'avère hétérogène, d'épaisseur très variable $(0,4$ à 2 cm) et fragilisé par des réseaux de fissures importants qui dateraient de sa conception (fig. 4). Il est sensible à l'eau froide dans laquelle il se dissout aisément. Plusieurs méthodes d'investigation complémentaires ont été nécessaires pour en déterminer la nature et comprendre le mode opératoire mis en ouvre lors de sa réalisation ${ }^{7}:$ microscopie optique (lumière blanche ou polarisée), microscopie électronique MEB (imageries en mode de contraste chimique, et analyses élémentaires

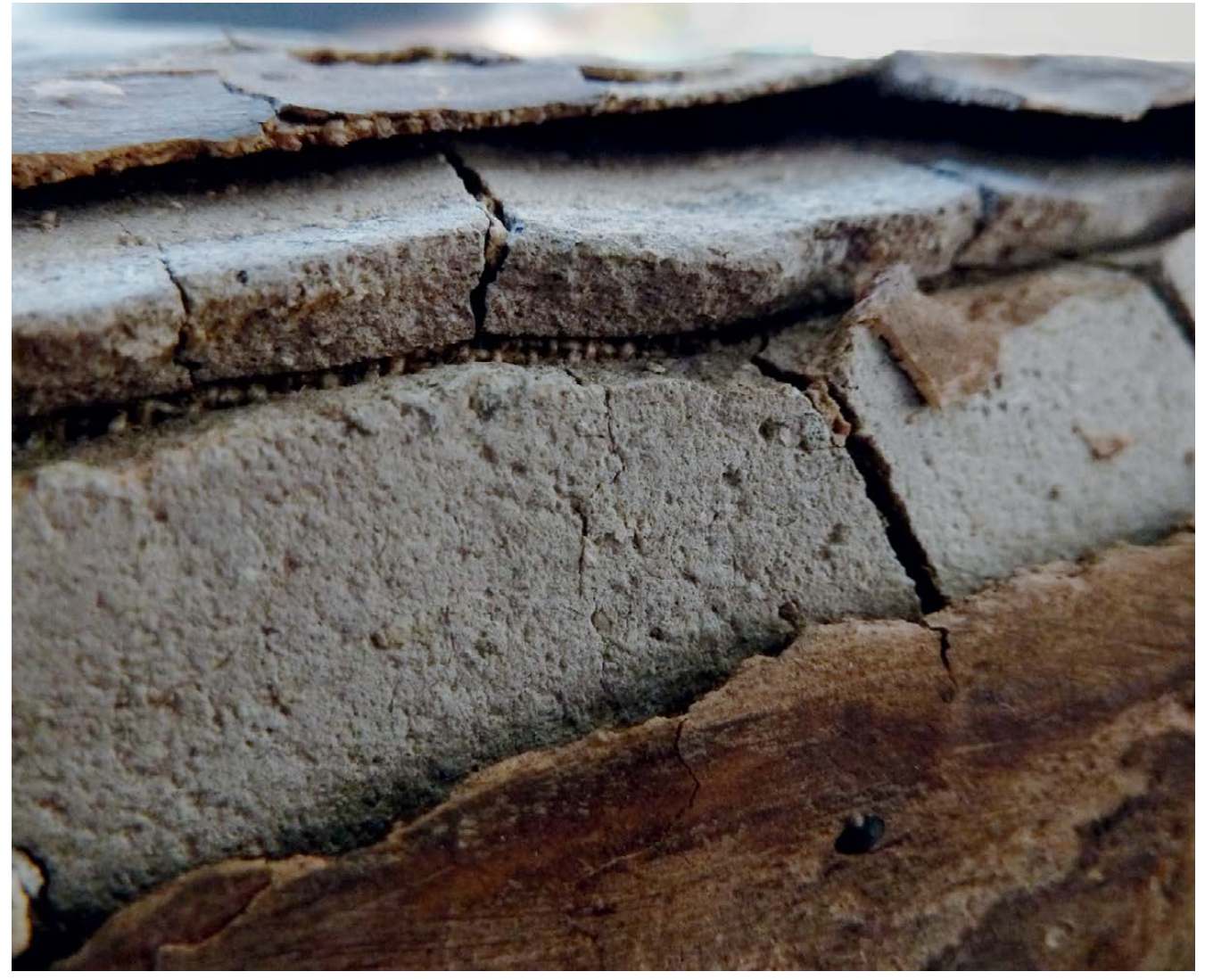

Fig. 4. Vue de l'état de fragmentation du mortier sur l'arrière de la jambe. (c) H. Gruau. 
semi-quantitatives en dispersion d'énergie de rayons $\mathrm{X}$ pour la composition des phases inorganiques), diffraction de rayons X pour identifier les phases cristallines et spectroscopie IRTF (Infra-rouge à transformée de Fourier) pour l'analyse des liants organiques et des colles.

\section{Analyse de la microtexture}

En section polie, ce mortier présente une texture plutôt fine, compacte et renfermant de très nombreuses inclusions de dimensions, morphologie, nature et couleur variables, ainsi que des fibres colorées (fig. 5 a et b et fig. 6). Des analyses élémentaires semi-quantitatives effectuées sur des aires jugées représentatives de la globalité du matériau $\left(\leq 2 \mathrm{~mm}^{2}\right)$ montrent une très nette prédominance de l'élément calcium (tableau 1). Ce mortier se caractérise par la présence de sulfates, de silicates et aussi de composés riches en potassium. La micro-analyse du ciment «matriciel » révèle un matériau dense formé d'imbrications complexes de " phases plâtreuses » difficiles à distinguer du fond, de gros grains lamellaires de gypse, de carbonates de calcium plus ou moins purs, de sulfates de calcium et de potassium, et de phases de nature indéterminée riches en magnésium pouvant provenir de la mise en œuvre d'un calcaire dolomitique. À cela s'ajoutent des quartz plus ou moins grossiers et divers silicates néoformés blanchâtres. Les inclusions brunes à rouges détectées regroupent des phases alumino-silicatées contenant un peu de fer (des argiles ?) et de rares petits fragments de matériau réfractaire proche de la brique. Enfin, les particules noires disséminées sont du noir de carbone ou des petits fragments de charbon de bois.

\section{Analyse cristallographique}

La nature des principales espèces cristallines qui constituent le mortier a été déterminée par diffraction des rayons $\mathrm{X}$. Ont été identifiés un sulfate de calcium dihydraté $\mathrm{Ca}\left(\mathrm{SO}_{4}\right) \cdot 2\left(\mathrm{H}_{2} \mathrm{O}\right)$ (plâtre), un sulfate double de calcium et de potassium $\mathrm{K}_{2} \mathrm{Ca}\left(\mathrm{SO}_{4}\right)_{2} \cdot \mathrm{H}_{2} \mathrm{O}$ (syngénite), et deux carbonates de calcium (calcite $\mathrm{CaCO}_{3}$ et monohydrocalcite $\mathrm{CaCO}_{3} \cdot \mathrm{H}_{2} \mathrm{O}$ ).

Un recoupement avec les micro-analyses a permis d'évaluer la proportion relative de ces quatre principaux constituants (fig. 7).

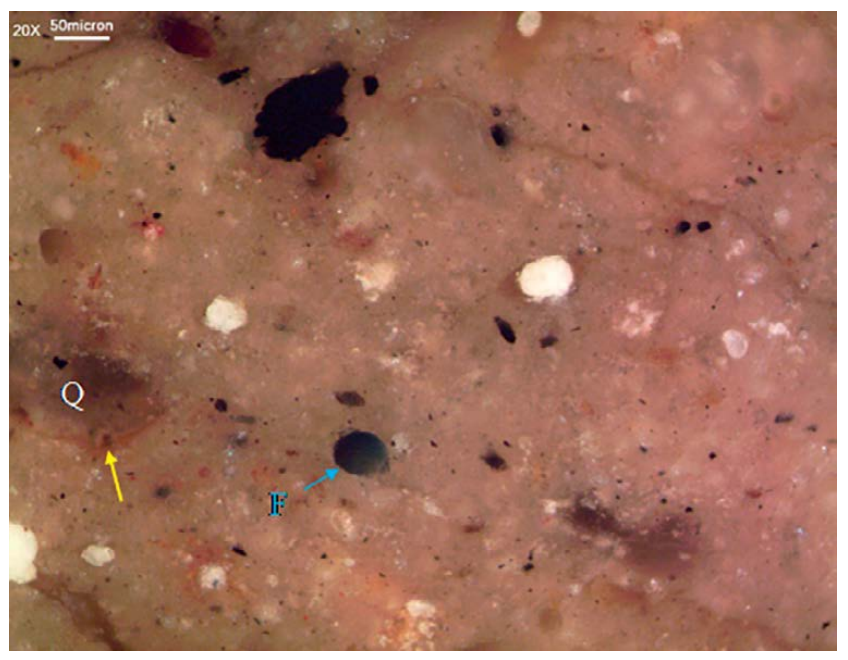

Fig. 5. Détail du mortier montrant un quartz Q et une fibre bleue F en section polie (microscope optique, barres d'échelle : 0,05 mm). (C) LAMOA.

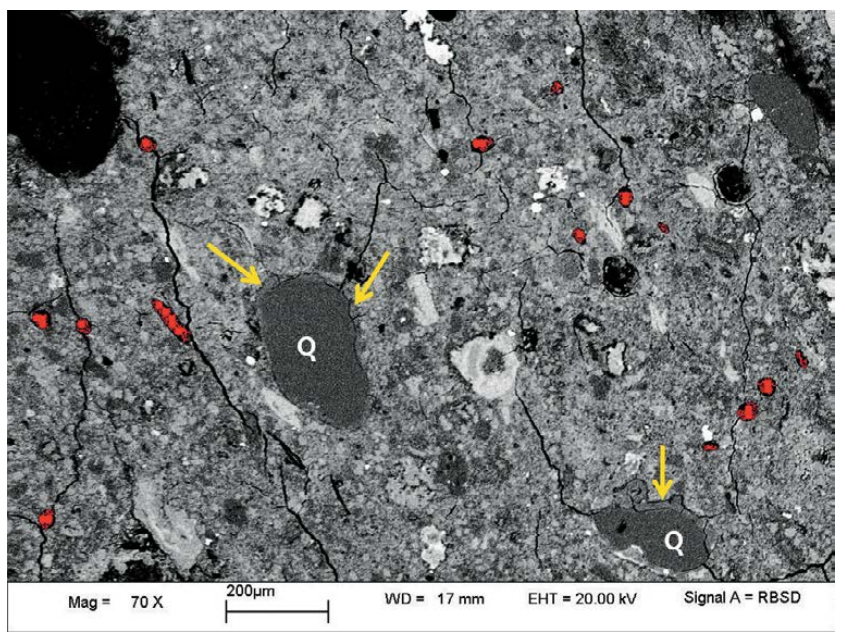

Fig. 6. Image chimique de la microtexture du mortier (MEB-ERD, barre d'échelle : 0,2 mm). Q = quartz $\left(\mathrm{SiO}_{2}\right)$ avec leur liseré périphérique réactionnel (flèches jaunes); en rouge = localisation des fibres organiques. (c) LAMOA.

Fig. 7. Évaluation sommaire par graphique à secteurs de la répartition des 4 espèces cristallines formant le mortier.

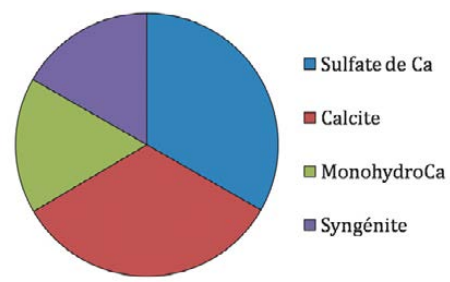

\begin{tabular}{|l|l|l|l|l|l|l|l|l|l|l|}
\hline $\mathbf{N a}_{2} \mathbf{O}$ & $\mathbf{M g O}$ & $\mathbf{A l}_{2} \mathbf{O}_{3}$ & $\mathbf{S i O}_{2}$ & $\mathbf{S}$ & $\mathbf{C l}$ & $\mathbf{K}_{2} \mathbf{O}$ & $\mathbf{C a O}$ & $\mathbf{F e}_{2} \mathbf{O}_{3}$ & $\mathbf{M n O}$ & Total : \\
\hline 2,3 & 5,8 & 2,9 & 13,9 & 15,4 & 0,8 & 8,3 & 50 & 0,5 & 0,2 & 100 \\
\hline
\end{tabular}

Tableau 1. Composition du mortier, moyenne arrondie des résultats des analyses semi-quantitatives par fluorescence X (MEB-EDX) exprimés en pourcentages d'oxydes élémentaires standards (sauf pour le soufre et le chlore) et normalisés à $100 \%$. 


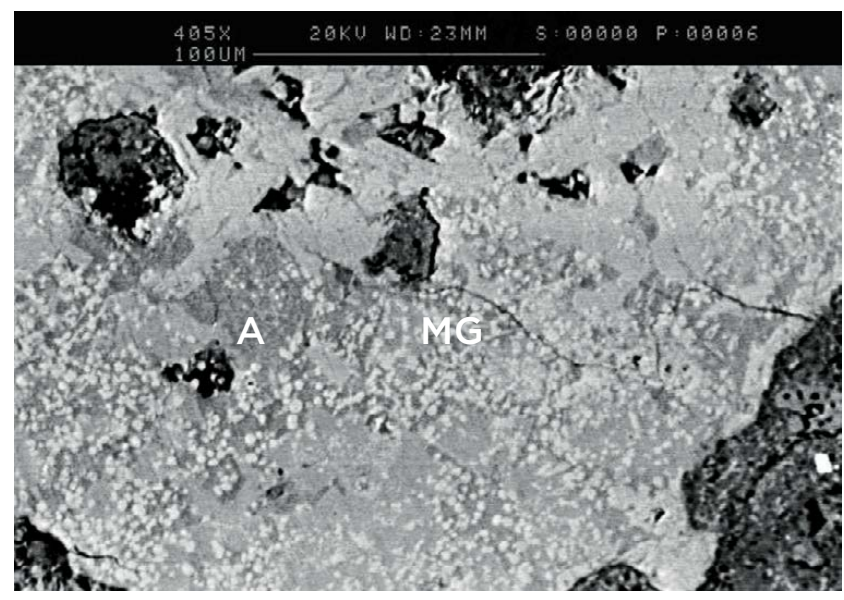

50 Fig. 8. Microtexture d'une zone réactionnelle polyphasée comportant de nombreux microgermes (MG) et des phases interstitielles « amorphes » (A) (MEB-ERD, barre d'échelle : $0,1 \mathrm{~mm})$. ( $\mathrm{LAMOA}$.

\section{Sur la nature des constituants majeurs}

À première vue, ce mortier s'apparenterait à un « plâtre modifié » dont la formulation diffère quelque peu des plâtres classiques, avec la formation de sulfates mixtes. La présence de potassium en particulier le rapprocherait des " plâtres alunés », à base d'alun de potassium connu comme accélérateur de prise. Cette particularité confère au mortier des propriétés probablement recherchées ici : une prise sans expansion ni retrait, une résistance à la traction et à la compression et aussi une dureté bien supérieures à celles du plâtre ordinaire ${ }^{8}$.

Les carbonates de calcium détectés dans ce mortier le rapprocheraient aussi des enduits à base de chaux de type " lime plaster » : cela suppose l'introduction d'une part de chaux dans le mélange de départ, qui va s'hydrater durant le gâchage puis subir le processus de carbonatation durant le séchage.

\section{Sur les phases néoformées et marqueurs technologiques}

Ce mortier comporte enfin une part significative de composés silicatés ayant plus ou moins réagi avec l'ensemble. En témoignent l'existence locale de microgermes de cristallisation alumino-silicatés, le développement d'agrégats polyphasés et aussi de phases interstitielles « amorphes » riches en calcium et silicium assurant une certaine cohésion à ce niveau (fig. 8). La microtexture des zones correspondantes n'est pas sans rappeler certains matériaux obtenus par frittage lors d'un chauffage. À cela s'ajoutent des grains de quartz émoussés dont la granulométrie varie de 150 à $300 \mu \mathrm{m}$ environ, indiquant qu'un sable fin à moyen a été associé au mélange de départ (fig. 6). Dans un plâtre, l'ajout de sables permet de lui conférer une certaine structure, rôle analogue à celui des dégraissants minéraux incorporés dans une pâte céramique. Dans un mortier, l'ajout de charges minérales limite le retrait, équilibre les tensions en cours de séchage et renforce sa dureté 8 .

On observe que ces grains de quartz comportent un liseré réactionnel périphérique d'une dizaine de microns d'épaisseur nettement enrichi en éléments issus du milieu réactionnel, et localement en éléments plus lourds (ex : fer). Ce phénomène incite à penser que le mélange quartz-mortier a pu subir ici une forme de chauffage. À travers la littérature concernant l'étude des mortiers anciens à la chaux, l'attention de certains chercheurs s'est portée sur l'amélioration des propriétés de ces matériaux en fonction de la température de l'eau de gâchage : elle ne devait pas excéder $100^{\circ} \mathrm{C}$ pour leur mise en œuvre et pouvait être le plus souvent comprise entre 71 et $93{ }^{\circ} \mathrm{C}^{8}$. De ce fait, en l'état des observations menées ici par le biais des analyses sur le mortier formant le corps du Christ, on pourrait pencher pour la séduisante hypothèse d'une eau de gâchage portée éventuellement à ébullition ou à une température assez proche.

\section{Les constituants organiques}

Ils sont de plusieurs natures : des particules éparses de noir de carbone et de charbon de bois, des fibres organiques colorées, et enfin un liant organique.

Diverses fibres ont été incorporées au mortier, majoritairement bleu clair ou foncé, brunes ou blanchâtres qui n'ont pu être identifiées précisément dans le cadre de cette étude (fig. 5 et 6). Néanmoins, les écailles observées sur certaines fibres bleues indiquent qu'elles sont d'origine animale et teintes, tandis que les brunes et les blanches sont d'origine végétale. Sur le plan technique, l'introduction de fibres renforce la cohésion et limite le «faïençage » ou le craquèlement ; celles-ci confèrent même une certaine flexibilité aux mortiers mis en œuvre dans les zones à risque ${ }^{9}$.

Enfin, une recherche de liant organique a été menée au moyen de la spectroscopie IRTF, après extraction dans l'eau froide puis séchage à $105^{\circ} \mathrm{C}$. Les bandes d'émission enregistrées à 3284, 2945, 1632, 1541, 1443-1405 et 1119-1091 $\mathrm{cm}^{-1}$ ont permis de révéler l'adjonction d'un liant protéinique.

$\mathrm{Au}$ final, les résultats des micro-analyses montrent une recherche très élaborée pour la conception de ce mortier, tant au niveau des constituants que de leur mise en ouvre, en vue de l'obtention d'un matériau suffisamment résistant pour une œuvre de ce format. Or les observations macroscopiques réalisées lors de la restauration et par le biais des images scanner montrent a contrario un matériau très fragmenté et qui semble avoir été endommagé dès sa conception. Le résultat escompté au moment de la fabrication n'aurait-il pas été obtenu? 


\section{La polychromie}

Une couche préparatoire de type gesso (à base de gypse) appliquée régulièrement sur le corps et le perizonium précède la réalisation de la polychromie. L'observation des couches picturales, sous loupe binoculaire puis grâce aux microanalyses, met en évidence une particularité au niveau des carnations : le dos et la face présentent deux polychromies originelles différentes, le dos ayant été exécuté en premier lieu. Les préparations à base de gypse sont comparables, mais les carnations diffèrent, tant dans leur couleur et leur composition que dans leur aspect final : rose pâle et gouttes de sang rouge vif au dos, et rose-beige avec des rehauts bleuâtres autour des plaies, gouttes de sang en relief et brillantes sur la face. Épaisse et striée à l'origine, la couche picturale de la face a été polie jusqu'à obtention d'un film fin, régulier et cassant, lui donnant un aspect satiné d'une grande qualité, typique des polychromies espagnoles ${ }^{10}$. En revanche, le perizonium, à rayures irrégulières bleues et rouges sur fond blanc, présente un aspect très mat qui vient apporter un contraste saisissant avec le poli des carnations.

Une autre particularité technique est à noter : la présence de plaies en relief sur l'abdomen et les genoux. De formes et de dimensions variées, elles sont réalisées selon une méthode

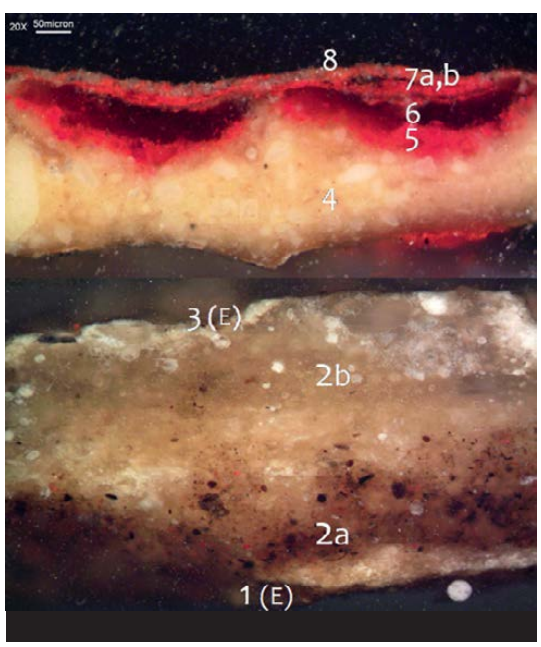

Fig. 10. Gouttes de sang du flanc droit. Microscope optique en lumière polarisée (barre d'échelle : 0,5 mm). Échantillon fractionné : 1 . Fin encollage au contact du tissu sous-jacent -2 . Préparation répartie sur deux niveaux (a : gesso altéré, b : plâtre) -3 . Fin encollage -4 . Carnation beige clair $-5.1^{\text {re }}$ couche rouge originale -6 . Couche de laque rouge ondulée $-7 \mathrm{a}, \mathrm{b}$. Repeint sur 1 à 2 niveaux -8 . Pellicule brillante (vernis?). (C) LAMOA. identique : une feuille de papier est placée entre les couches de préparation, puis découpée et évidée en son centre ; les bords sont ensuite relevés de manière à figurer les chairs de la plaie, puis l'ensemble est peint (fig. 9).

D'après les analyses pigmentaires, les carnations rose pâle du dos sont à base de blanc de plomb et contiennent de fines particules disséminées d'ocre, de silicates de fer et de noir de carbone, avec de rares grains orangés très riches en plomb (minium ?). On y détecte un liant protéinique de type gélatine ou caséine. Les carnations rose-beige de la face sont constituées d'une première couche formée d'un mélange de blanc de plomb, de charges calciques (calcite et gypse) et de particules brun-rouge d'ocre et de silicates de fer, couche recouverte d'un très fin lavis rougeâtre dont la composition est voisine de celle des carnations du dos.

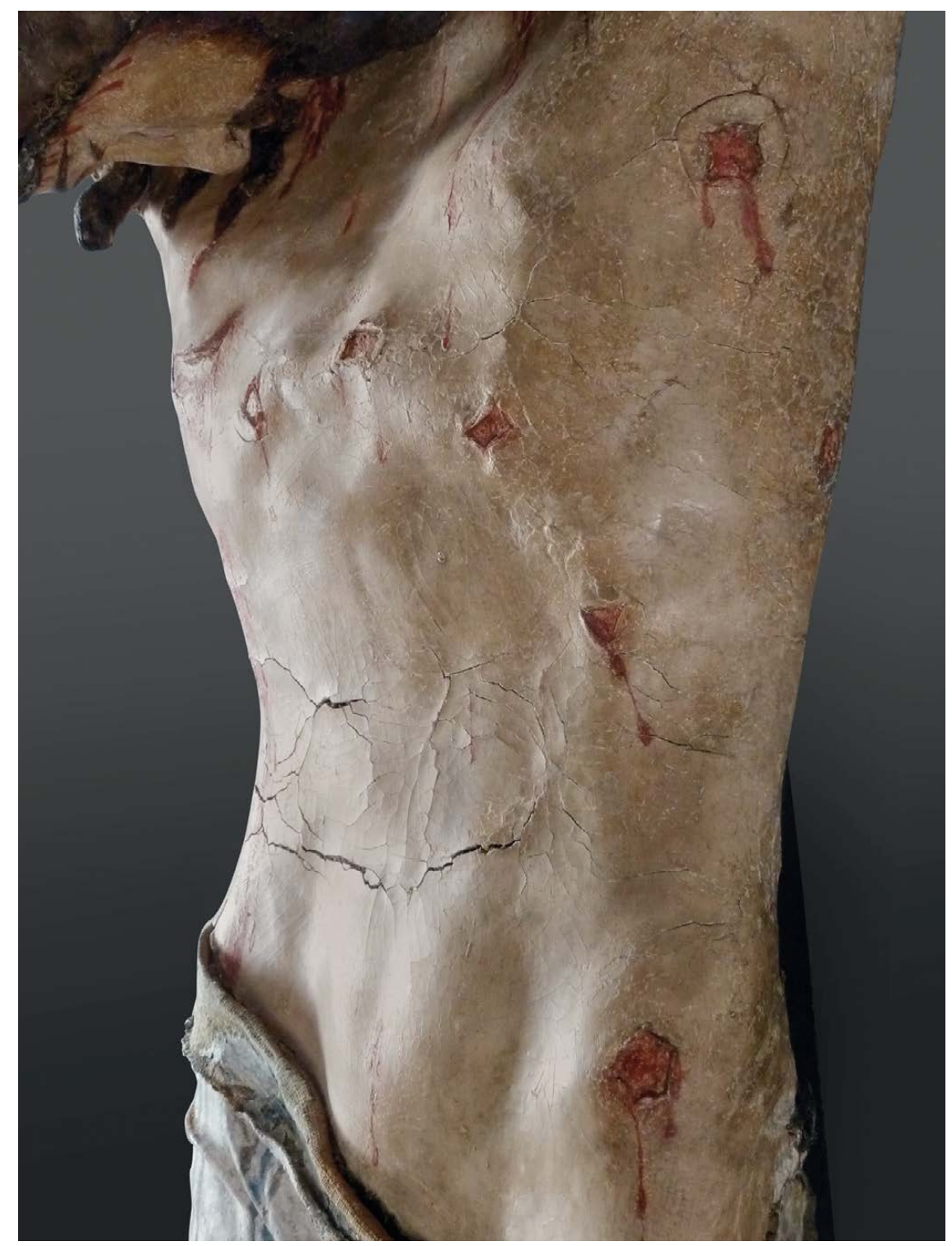

Fig. 9. Détail des plaies en relief. () H. Gruau. 
La plaie sanguinolente du flanc droit du Christ (fig. 10) est traitée en dégradé de rouges sur un fond blanc mêlant du blanc de plomb et des charges calciques. Elle est composée d'une couche picturale rouge à rouge-rosé associant blanc de plomb, vermillon et un pigment rouge organique laqué, recouverte d'une couche de laque rouge plus ou moins épaisse et ondulée en section. L'ensemble est surmonté d'une phase de repeint comportant une à deux fines couches rouges à base de blanc de plomb et de pigments riches en fer.

Le décor du perizonium est formé de rayures irrégulières bleues et rouges sur fond blanc, posé sur l'enduit de type gesso. Les rayures bleues du décor contiennent du pigment bleu de smalt qui présente des phénomènes d'altération typiques des matériaux vitreux hydratés. Les rayures rouge-orangé renferment des sulfures d'arsenic altérés ; les analyses tendent à indiquer que le pigment d'origine serait du réalgar rougeorangé affecté par des phénomènes de jaunissement de type pararéalgar et, localement, par un noircissement en association avec des sels de plomb. Sous ce décor de rayures, la présence locale de feuilles d'or sur mixtion ne manque pas de surprendre : faut-il l'assimiler à un essai ou à un repentir, ce qui appuierait l'idée d'une ouvre expérimentale ${ }^{11}$ ?

Enfin, la chevelure du Christ comporte un niveau de polychromie original complexe en trois strates : une dorure partielle (comparable à la présence d'or sous le décor rayé du perizonium, et qui pose les mêmes interrogations) sur un bol rouge continu, une matière organique noirâtre avec des traces de manganèse, et enfin une couche picturale marron foncé à base de terres d'ombre, d'ocre et de laque brune, intimement liée à la polychromie originale des carnations (face), qu'elle recouvre en décors de mèches peintes. L'ensemble est surmonté d'un premier repeint partiel formé d'une série de trois niveaux bruns à noirs et reflets rouges, riches en pigments au fer et manganèse, puis d'une phase de retouche croûteuse gris foncé, carbonatée et d'aspect laiteux.

La possibilité d'un niveau original antérieur doit être considérée, mais ne trouve aucune cohérence lors de l'observation de l'ensemble de la polychromie, au regard des concordances entre les carnations, cheveux et perizonium et de l'étendue des surfaces concernées.

\section{Rapprochement du Christ de Nantes avec les modèles espagnols et mexicains}

À l'occasion de sa restauration, le Christ de la cathédrale de Nantes, jusqu'alors méconnu, a fait l'objet d'une totale redécouverte : en effet, les recherches documentaires, ainsi que l'expertise du musée du Louvre ${ }^{12}$ qui a souligné la rareté de cette œuvre à l'échelle nationale, ont permis de mettre en lumière l'intérêt historique et artistique de cette sculpture atypique. Une provenance latino-américaine a été évoquée, mais l'origine hispanique, mentionnée à plusieurs reprises dans les archives conservées, n’a jamais été remise en question.
Cette œuvre se distingue par la silhouette étonnante du Christ, la tête totalement affaissée sur le torse, ne laissant deviner que quelques traits de son profil. Avant la restauration, cette position particulière avait inquiété les conservateurs, qui pensaient qu'une faiblesse structurelle pouvait compromettre la conservation de l'objet sur le long terme. L'analyse stylistique a révélé que le Christ de Nantes appartient à la typologie du «Christ de Burgos », l'un des crucifix les plus vénérés du monde hispanique : connu par des images remontant au XIV ${ }^{\mathrm{e}}$ siècle, le «Christ de Burgos » constituait un support de dévotion pour les fidèles, mais également une statue miraculeuse qui possédait le pouvoir de guérison et faisait l'objet d'un culte fervent, proche de celui traditionnellement dévolu aux reliques. Les traces de ce rôle cultuel se lisent d'ailleurs sur la sculpture de Nantes : lors de sa restauration, des marques de grattage ont été observées sur les plaies, qui sont autant de signes de dévotion intentionnels. Le succès de cet archétype s'est répandu à travers le monde entier, donnant lieu à l'émergence de nombreux cultes locaux établis dans toute l'Espagne, en Flandres et en Amérique latine.

Témoignage de la diffusion de ce modèle à travers l'Europe, le Christ de la cathédrale de Nantes s'inscrit dans la mouvance doloriste de l'art espagnol ${ }^{13}$, qui représente avec un réalisme aigu la souffrance du Christ sur la croix.

Si l'origine sud-américaine du Christ de Nantes s'est rapidement révélée erronée, la confrontation technologique avec les Christ de maïs mexicains a en revanche été fructueuse. La production de "sculptures légères » destinées aux processions est très importante aux $\mathrm{XVI}^{\mathrm{e}}$ et $\mathrm{XVII}^{\mathrm{e}}$ siècles au Mexique, afin de répondre aux nombreuses demandes des couvents franciscains et augustins des régions de México et de Michoacán ; les matériaux utilisés varient (pâte de maïs, tiges de maïs, papier d'amate à base de fibres de ficus, papiers européens), mais les modes de fabrication issus des traditions précolombiennes et notamment du savoir-faire des plumassiers ${ }^{14}$ sont assez constants. Les Christ de maïs sont des œuvres de mieux en mieux documentées grâce au travail conjoint de restaurateurs et de chercheurs ${ }^{15}$, et la complexité du sujet s'étoffe au fur et à mesure de l'avancée des connaissances. Ces représentations répondent à un but précis : le souci de légèreté en vue des processions. Les œuvres sont creuses en totalité ou en partie, et leur matière constituante, obtenue par estampage dans un moule à bon creux est renforcée à l'intérieur par des papiers encollés. Des éléments en bois de colorín (bois d'Erythrina) sont également mentionnés, permettant une armature interne et jouant le rôle d'ossature fonctionnelle et peut-être symbolique. Certaines parties, telles les mains ou les pieds, pouvaient également être sculptées dans le bois de colorín. Les membres sont réalisés séparément puis assemblés, et les volumes retravaillés : ajout ou retrait de matière par modelage ou par reprise sculptée. Le positionnement des bras et de la tête pouvait varier au moment du montage, permettant une diversité dans les modèles produits. Les perizonium peuvent être réalisés en papier, modelés autour du corps, puis polychromés ${ }^{16}$. L'exportation de certaines de ces sculptures légères vers 
l'Europe et, plus précisément, l'Espagne est avérée : au moins six exemples de Christ légers y sont connus à ce jour, mais d'autres modèles non identifiés peuvent exister. En effet, la vocation déambulatoire de ces objets de procession les rend particulièrement vulnérables (intempéries, manipulations régulières) et ils ont pu être délaissés lorsque trop altérés. D'autre part, il est parfois difficile de connaître la matérialité de ces œuvres lorsqu'elles ne peuvent être approchées et observées de près, le Christ de la cathédrale de Nantes en est la preuve.

Le Christ de Nantes est techniquement comparable en bien des points à ces Christ de procession. Sa spécificité se situe dans les matériaux utilisés : le mortier remplace la pâte de maïs, les textiles encollés se substituent au papier encollé, et le bois de colorín mexicain est ici remplacé par le liège espagnol dans les mains et les pieds. La différence majeure résulte cependant dans le poids final de l'objet : lorsque les Christ de maïs ne pèsent qu'une dizaine de kilos, le Christ de Nantes affiche, pour une taille comparable, un poids de 28 kilos sans la croix.

L'hypothèse d'une influence mexicaine, ou tout au moins la connaissance de ces modèles de sculptures légères issues des savoir-faire précolombiens, est tentante. Le résultat, trop lourd et fragile, aurait-il été jugé comme un échec et la réalisation d'autres modèles abandonnée ? En l'absence d'œuvres comparables techniquement, qui attesteraient l'existence d'une « série » comme on peut l'observer dans le cas des Christ de maïs, cette hypothèse ne peut être entièrement écartée.

\section{État de conservation actuel et interventions passées}

L'aspect sombre et empoussiéré, les zones de cassure, de déchirure, la fragilité structurelle sont autant de raisons qui ont conduit à l'étude, puis à la restauration de cette œuvre. Plusieurs restaurations antérieures ont ainsi été identifiées, la dernière pouvant être associée au déplacement du Christ vers la cathédrale Saint-Pierre de Nantes. La croix est un élément de réemploi adapté en longueur à la taille du Christ ; le mode de fixation originel (pièce de métal cuivreux insérée au sein du mortier constitutif) a cependant été conservé et demeure opérationnel.

D'un point de vue structurel, les principales altérations sont les suivantes : les deux bras sont fragmentés, l'un au niveau du coude, l'autre au niveau de l'épaule, il existe des manques de matière importants sur la chevelure, les assemblages de mortiers se sont ouverts (torse, jambes) et certains textiles se sont déchirés ou déformés (cas du perizonium).

De nombreux bouchages ont été réalisés selon deux campagnes successives de restauration ; souvent maladroits et épais, ils ont contribué à alourdir la structure et à la fragiliser. Une armature métallique a été placée au dos afin de pallier la mobilité des bras : en fer plat, fixée dans le mortier des bras et du dos au moyen d'une trentaine de clous forgés puis recouverte de textiles encollés, cette restauration a grandement participé à la fracturation du mortier interne. La main droite, refaite, a été fixée au bras par un entrelacs de fils métalliques.

La polychromie a fait l'objet de deux repeints, appliqués localement sur les zones endommagées et les zones d'usure. Sur ces dernières, nous devons distinguer les usures naturelles de celles relevant de la pratique de dévotion déjà évoquée. Les retouches des campagnes de restauration successives ont principalement servi à masquer l'impact de ces marques de dévotion.

Outre l'aspect sombre, terne et très empoussiéré de la surface, la polychromie présente de multiples altérations : soulèvements, lacunes, craquellements, déformations d'écailles sur le dos du Christ. Certaines des plaies en relief ont été comblées par les bouchages (genoux, épaule senestre) et les décors ont été modifiés : des gouttes de sang originales ont été masquées et, a contrario, des plaies ont été ajoutées. Masqué par l'encrassement général et les repeints, le contraste entre l'aspect satiné des carnations et la matité du perizonium n'est plus perceptible. Enfin, les méthodes de nettoyage inadaptées des interventions antérieures ont endommagé irrémédiablement certaines zones des carnations.

\section{La restauration}

Suite au traitement curatif insecticide par anoxie statique mené en atelier ${ }^{17}$, la restauration a consisté en premier lieu à retirer toutes les anciennes interventions (armatures métalliques et textiles non originaux, bouchages inadaptés). Le travail sur la polychromie a ensuite été entrepris : fixage des écailles au moyen de résines adaptées aux différentes problématiques $^{18}$, puis retrait mécanique des repeints au scalpel (fig. 11) et nettoyage de la surface. La grande fragilité des carnations et leur réactivité aux solvants ont été prises en compte, et le choix des produits ainsi que le geste du restaurateur ont été constamment adaptés lors de cette opération afin de respecter au mieux l'aspect initial satiné des chairs ${ }^{19}$. Sur certaines zones, la surface est irrémédiablement endommagée, mais le " poli » des carnations a cependant pu être remis en valeur sur la majorité de la surface, et le contraste avec la matité du perizonium est de nouveau apparent (fig. 12).

Le remontage structurel a été ensuite envisagé. L'épaule gauche, entièrement déformée et fragmentée en son mortier interne, est tout d'abord reconstruite : les textiles rigidifiés par l'épaisse colle animale sont ramollis par compresses d'eau chaude puis remis en forme et réappliqués de manière à être à nouveau en contact avec la surface du mortier. L'ensemble retrouve sa cohésion, mais un manque de matière important ${ }^{20}$ demeure sous le textile polychromé : une cale est confectionnée, qui sert ensuite de support et permet la réapplication du textile.

Le remontage du bras droit, fractionné au niveau du coude, est effectué au moyen de deux chevilles en hêtre venant coulisser dans des fourreaux placés sur la paroi 


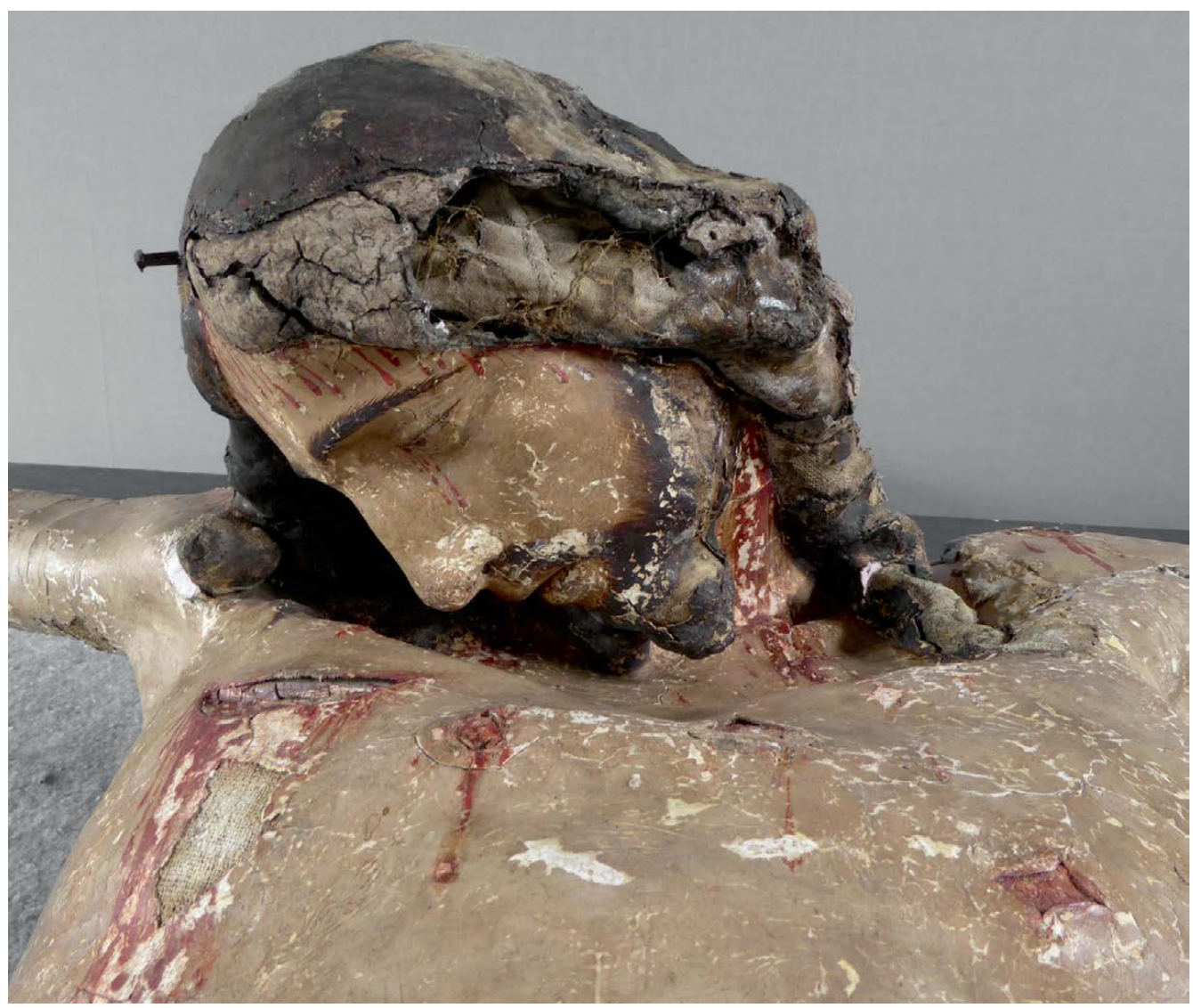

Fig. 11. Détail après retrait des repeints. (C) H. Gruau.

interne du bras, assemblage démontable si besoin. Les clous de la crucifixion, datant de la mise en place du Christ sur la croix de réemploi, sont adaptés afin de permettre un démontage ultérieur aisé.

La réintégration des manques a fait l'objet de nombreuses discussions collégiales afin de réaliser les choix les mieux adaptés à l'intérêt historique et technique de cette œuvre, ainsi qu'aux impératifs structurels liés à sa conservation et à sa présentation. Les manques relatifs aux assemblages entre les éléments constitutifs ont ainsi été comblés en premier lieu ; les doigts et orteils devenus lacunaires suite à la dépose d'anciennes restaurations ont été restitués, leur volume étant dicté par la présence d'éléments originaux. Cette condition n'était en revanche pas applicable à l'importante lacune constituée par la chevelure ; il a donc été décidé de ne pas intervenir sur cette zone.

Des retouches colorées ${ }^{21}$ poussées ont été réalisées sur les zones de bouchage, tandis que les zones grattées par dévotion ont été simplement intégrées par retouches colorées légères, sans remise à niveau préalable afin de conserver le témoignage de cette pratique votive particulière. Enfin, le textile du perizonium a fait l'objet d'un traitement par une restauratrice spécialisée ${ }^{22}$ qui a stabilisé les bordures du tissu.

\section{Conclusion}

Le travail d'investigation, de recherches scientifiques, l'apport de la tomodensitométrie, les comparaisons stylistiques et techniques avec des œuvres espagnoles et mexicaines nous ont permis de mieux comprendre cette ouvre énigmatique et de la restaurer avec tous les soins nécessaires.

Des hypothèses ont pu être avancées, mais certaines questions restent encore en suspens : s'agit-il d'une œuvre expérimentale, travail d'artistes espagnols inspirés par leurs confrères mexicains, n'ayant pas donné suite à la fabrication d'autres exemplaires en raison du poids trop élevé et de la fragilité du mortier ? Existe-t-il d'autres Christ de procession mettant en scène les mêmes matériaux et techniques de fabrication ? Le moule «à bon creux » utilisé pour l'estampage du mortier a-t-il pu servir à la confection d'autres œuvres, à l'instar des Christ de maïs mexicains? Quel modèle a servi pour 


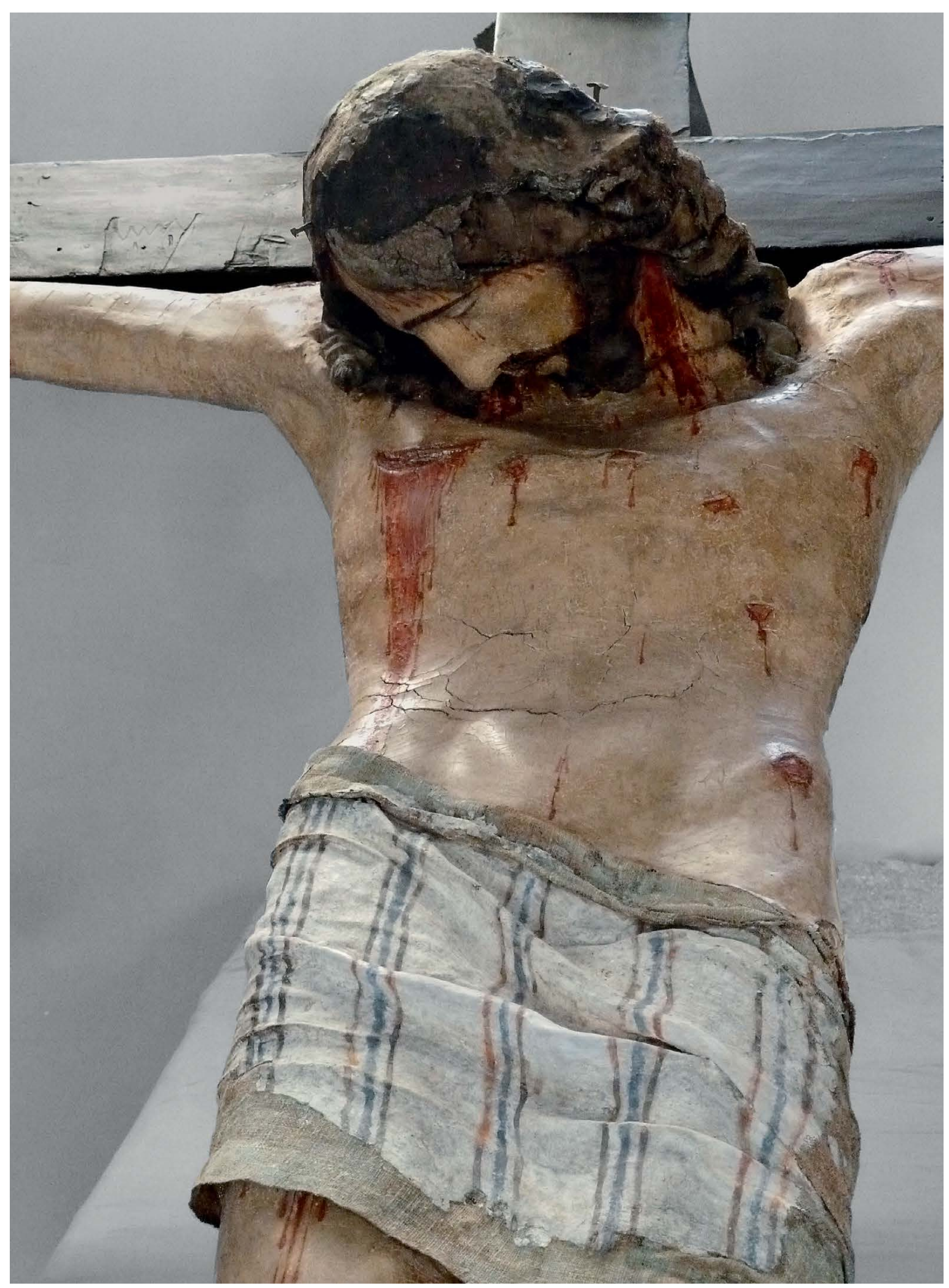


la prise d'empreinte du moule initial ? Toutes ces questions sont un point de départ à une recherche qui pourrait être élargie à un contexte plus ample, tant géographique que stylistique, et qui permettrait de rattacher cette sculpture à un corpus d'œuvres similaires.

De 2009 à 2015, la Conservation régionale des monuments historiques a lancé et financé la restauration de cet objet exceptionnel, participant à la redécouverte de la richesse du patrimoine mobilier conservé à l'intérieur de la cathédrale de Nantes. Cette sculpture, jusqu'alors ignorée, a suscité un véritable engouement de la part de tous les partenaires réunis autour de ce chantier de longue haleine (conservateurs, restaurateurs, scientifiques, chercheurs, affectataires). Cet intérêt grandissant a également abouti à la reconnaissance de sa valeur patrimoniale, qui s'est traduite par un classement au titre des monuments historiques en juin 2014. Le 18 décembre 2015, le Christ a été réinstallé, à la demande de l'évêque, dans la nef sur le pilier faisant face à la chaire à prêcher. La qualité et la subtilité de cette œuvre sont désormais de nouveau accessibles aux fidèles et visiteurs de la cathédrale.
Notes

1. Extrait du livre paroissial SaintPierre de Nantes, 1907-1990, archives historiques du diocèse de Nantes. Cote :

P. St Pierre A7, année 1978.

2. Grenouilleau, 2012

3. Gruau, 2015.

4. Les manques de matière ne nous permettent pas de déterminer précisément le découpage des parties au niveau de la chevelure, au-dessus du front.

5. Technique utilisée sur l'Ecce Homo, Gregorio Fernandez ( $\mathrm{XvI}^{\mathrm{e}}$ siècle), musée diocésain de Valladolid ; Exp. Londres, 2010, p. 130-135.

6. Aujourd'hui manquante, cette couronne pouvait consister en branches tressées.

7. Etcheverry, 2014.

8. Elert, 2002.

9. Bennett, 2002.

10. Exp. Londres, 2009, p. 58-71.

11. Le décor de rayures apparaît comme le niveau original destiné à être vu et ne peut être confondu avec un repeint ; la comparaison des couches préparatoires lors des micro-analyses appuie cette interprétation.

12. Kientz, 2013.

13. Exp. Londres, 2009.

14. Dehouve, 1997.

15. On peut citer notamment les travaux menés par Rolando Araujo Suarez sur le Christ de Chrurubusco, Mexico, et la restauration du Christ de la Basílica de San Juan Batista de Telde, Gran Canaria, par Pablo Francisco Amador Marrero et Carolina Besora Sanchez.

16. Cristo de Telde, Gran Canaria.

17. Traitement réalisé et contrôlé par Aurélie Fortin (entreprise 3PA, La-Croix-enTouraine, 37), consistant à éliminer les insectes xylophages à tous les stades (insecte adulte, larve et œuf) par privation d'oxygène.
18. Le Paraloïd B72 ${ }^{\circledR}$ a été choisi dans le cas de soulèvements entre le gesso et les écailles très fines des carnations, en raison de sa fluidité (concentré à $10 \%$ dans de l'acétate d'éthyle). Lorsque les soulèvements se situaient à l'interface textile - gesso, le Primal E330 ${ }^{\circledR}$ en solution à $5 \%$ dans de l'éthanol a été utilisé.

19. Suite à un premier nettoyage au White Spirit ${ }^{\circledR}$, un complément de nettoyage à l'eau tiède (coton roulé sur la surface) ou eau additionnée de tensio-actif (Triammonium Citrate à $3 \%$ ) est réalisé, adapté aux différentes zones. L'éthanol permet de supprimer les reliquats des anciens fixatifs. La polychromie mate du perizonium est nettoyée par gommage (Whisab ${ }^{\circledR}$ ), puis reprise ponctuellement à l'éthanol.

20. Il s'agit probablement d'un défaut d'origine.

21. Couleurs de conservation Gamblin ${ }^{\circledR}$.

22. M. F. Levoir, restauratrice de textiles, Nantes.

\section{Bibliographie}

Amador Marrero P. F., Besora Sanchez C., 2000, «Aportaciones al estudio de los Cristos tarascos en Canarías. El ejemplo del Santissimo Cristo del Altar Mayor de la basílica menor de San Juan Bautista de Telde, GranCanarías ", III Coloquio de historia de Canario-America, https://dialnet.unirioja.es/servlet/ articulo? codigo $=2200209$.

Araujo Suarez R., 1989, «El Cristo de Churubusco, Conservación de Esculturas de papel amate y caña de maíz ", Cuaderno Tecnicos, Mexico.

Arquillo Torres J., Morales Mendez E., 2010, «La industralización de las esculturas religiosas en la nueva España : Cristos de caña de maíz ", Actas del X congreo internacional de rehabilitacion del patrimonio arquitectonico, CICOP Chile.

Bennett E., 2002, "Lime plaster and render reinforcement", Cathedral Communications Limited, www. buildingconservation.com.
Dehouve D., 1997, « Le Christ et le plumassier ", Le Christ et le Plumassier en Nouvelle-Espagne au XVI siècle, ENS Édition, Paris, t. II, p. 322-334.

Elert K., Rodriguez-Navarro C., Pardoe S., Hansen E., Cazalla O., 2002, "Lime mortars for the conservation of historic buildings", Studies in Conservation 47, p. $62-75$.

Exp. Londres, 2009, The sacred made real, Spanish painting and sculpture 1600-1700, [Cat. Exp. London, National Gallery of Art, 2009], Bray X. (dir.), p. 58-71 et p. $130-135$.

Guttierez J., 2013, «Un Christ espagnol à la cathédrale de Nantes ", 100 ans de monuments historiques en Pays de la Loire, Parlez-moi patrimoines... DRAC Pays de la Loire, Nantes.

Kientz G., 2013, « Un Christ en croix d'origine hispanique », La grâce d'une cathédrale, Éditions de la Nuée Bleue, Strasbourg, p. 231.

Documents inédits

Etcheverry M.-P., 2014, «Étude de prélèvements polychromes provenant du Christ en croix de la cathédrale de Nantes ", Le mortier-support (Vol. I, 23 p.), La polychromie (Vol. II, 67 p.), LAMOA Expertise, rapports d'étude LAM1410-54 non publiés, DRAC Pays de la Loire, Conservation régionale des monuments historiques, Documentation des objets mobiliers, Nantes.

Grenouilleau C., 2012, Étude diagnostic d'un Christ en croix, rapport d'étude, DRAC Pays de la Loire, Conservation régionale des monuments historiques, Documentation des objets mobiliers, Nantes.

Gruau H., 2015, Rapport d'étude et de restauration, Christ en croix, Cathédrale Saint-Pierre de Nantes, DRAC Pays de la Loire, Conservation régionale des monuments historiques,

Documentation des objets mobiliers, Nantes. 